\title{
Civilisations
}

Revue internationale d'anthropologie et de sciences

humaines

$51 \mid 2004$

Religions transnationales

\section{Les Assemblées de Dieu du Burkina Faso en contexte}

A propos de : P.-J. LAURENT, Les pentecôtistes du Burkina Faso. Mariage, pouvoir et guerison, Paris, Karthala, 2003

Joël Noret

\section{OpenEdition}

\section{Journals}

Édition électronique

URL : http://journals.openedition.org/civilisations/692

DOI : $10.4000 /$ civilisations. 692

ISSN : 2032-0442

\section{Éditeur}

Institut de sociologie de l'Université Libre de Bruxelles

\section{Édition imprimée}

Date de publication : 1 janvier 2004

Pagination : 171-181

ISSN : 0009-8140

\section{Référence électronique}

Joël Noret, «Les Assemblées de Dieu du Burkina Faso en contexte », Civilisations [En ligne], 51 | 2004 ,

mis en ligne le 07 janvier 2009, consulté le 30 avril 2019. URL : http://journals.openedition.org/

civilisations/692 : DOl : 10.4000/civilisations.692

Ce document a été généré automatiquement le 30 avril 2019.

(c) Tous droits réservés 


\title{
Les Assemblées de Dieu du Burkina Faso en contexte ${ }^{1}$
}

\author{
A propos de : P.-J. LAURENT, Les pentecôtistes du Burkina Faso. Mariage,
} pouvoir et guerison, Paris, Karthala, 2003

Joël Noret

\section{RÉFÉRENCE}

P.-J. LAURENT, Les pentecôtistes du Burkina Faso. Mariage, pouvoir et guerison, Paris, Karthala, 2003.

1 La présence des Assemblées de Dieu (AD) en Afrique de l'Ouest dans la deuxième décennie du XXe siècle et leur développement progressif en pays mossi à partir de 1921 illustrent à merveille l'implantation extrêmement rapide du pentecôtisme en Afrique, presque contemporaine de la naissance même du mouvement; le phénomène a déjà été souligné de nombreuses fois. Là n'est pas, toutefois, l'objet principal de l'ouvrage dont il va être question ici, même si on y trouve une partie historique d'une quarantaine de pages, dont une vingtaine consacrées à l'histoire proprement dite de l'Eglise au Burkina Faso.

2 L'étude de Pierre-Joseph Laurent, fruit d'une dizaine d'années de fréquentation des Assemblées de Dieu burkinabées, propose plutôt, d'une manière générale, une compréhension de celles-ci qui fait largement appel au contexte social dans lequel elles se sont développées, surtout depuis le milieu des années 1980. A de nombreuses reprises, l'auteur nourrit en effet son étude d'analyses plus générales du contexte social national ou africain, qui éclairent utilement l'objet principal de l'ouvrage. C'est pour souligner cette attention soutenue au contexte qu'a été choisi le titre de cette note, mais c'est aussi parce que je souhaite, dans la seconde partie de ce texte, rebondir sur certains éléments $\mathrm{du}$ contexte transnational dans lequel dès le début se sont trouvées inscrites les Assemblées de Dieu du Burkina Faso. En effet, sur ce contexte, l'exposé de l'auteur souffre de certaines imprécisions et de certains manques d'une part, et peut faire l'objet de prolongements intéressants d'autre part. 
3 Exemplaire tout d'abord du souci du contexte social «local», que l'auteur expose avec une maîtrise indéniable, est l'analyse qu'il propose de la mise en place du mariage pentecôtiste. Les transformations de la famille étendue et de l'institution lignagère sont en effet au centre des évolutions qu'accompagnent les développements des Assemblées de Dieu en milieu rural mossi, et l'auteur montre bien comment l'alliance pentecôtiste, permise par la protection d'un "Dieu de puissance », se présente comme un produit synthétique qui intègre subtilement les différentes tensions parcourant la société et la famille mossi tout en continuant à pouvoir se justifier bibliquement: «le mariage protestant tente de régler, à travers une alliance qui se voudrait définitive, à la fois la quête du libre choix du conjoint, les impératifs lignagers et la production d'alliés » (p. 99). L'ambiguïté du rôle des pasteurs, de leur attitude envers la liberté proclamée de choix du conjoint d'une part, et des relations entre pasteurs et chefs coutumiers d'autre part, est ici parfaitement bien analysée et illustrée. Les pasteurs récupèrent en effet certains aspects du statut des chefs de lignage à travers leur rôle de médiateurs dans les affaires d'alliances, et il peut dès lors être tentant pour eux de chercher à orienter plus ou moins fortement les alliances plutôt que de "simplement » les encadrer religieusement. Enfin, le cas du mariage permet aussi de repérer, au niveau de l'Eglise dans son ensemble, une oscillation entre un mode de fonctionnement intransigeant lors, par exemple, des campagnes d'évangélisation (massivement enga-gées dans la démonisation de l'univers de la coutume), et un autre beaucoup plus pragmatique, faisant davantage de place à la négociation, lors des mariages et de la conclusion d'alliances.

4 Tout au long de l'ouvrage, l'auteur observe également la mise en place d'un nouvel ethos (notamment à travers la figure du " pasteur-paysan », qui jouera un si grand rôle dans les développements de l'Eglise en milieu rural), plus individualiste, même s'il conserve l'hétéronomie du sujet mais en déplace le fondement : celui-ci n'est plus l'ensemble formé par le lignage et les ancêtres mais un Dieu derrière la puissance de qui les communautés pentecôtistes peuvent se construire sans devoir trop craindre la jalousie (et les actes potentiels de malveillance qu'elle est supposée nourrir) des milieux sociaux (en particulier lignagers) que la conversion de chacun lui a permis de mettre à distance. Et un autre mérite de l'ouvrage est précisément d'insister sur la dimension éthique et psychologique de ces nouveaux espaces sociaux que constituent les assemblées pentecôtistes, dans le sens où l'auteur montre bien que la conversion s'inscrit dans une sorte de quête d'apaisement. On doit ainsi pouvoir rentrer dans un rapport à l'Autre davantage pacifié, dans un contexte décrit comme celui de la " modernité insécurisée ».

Relevant de manière originale et certainement très pertinente que la crise du lien social (qui renvoie à une forme de crise de l'institution) dans la société burkinabée actuelle provient de la difficulté à établir ou à restaurer des relations pacifiées entre les individus et entre les groupes, l'auteur fait surtout remarquer que c'est dans ce contexte de "modernité insécurisée », où les individus sont pris entre les réalités persistantes de la dépendance communautaire et leurs aspirations à un mode de vie et à des trajectoires d'accumulation plus individualisées, que les Assemblées de Dieu se montrent capables, d'une part, d'offrir des ressources de gestion des forces de l'invisible qui prennent de plus en plus de place dans la régulation de la vie sociale, et présentent, d'autre part, l'opportunité de s'inscrire dans un nouveau réseau socio-religieux (celui de l'église locale, et, d'une manière plus générale, de l'ensemble des "convertis »), où les relations se présentent précisément a priori comme apaisées. En suivant ce succès des Assemblées de Dieu dans la société burkinabée, P.-J. Laurent décrit aussi, en quelque sorte, un cas de 
montée en puissance du religieux comme principe de structuration de la société (tant au plan cognitif, avec un travail pentecôtiste de l'imaginaire tout à fait important, qu'au plan de l'organisation sociale) en régime de modernité africaine, où la Bible devient un véritable « mode d'emploi du monde».

tMais P.-J. Laurent évoque aussi les évolutions internes des Assemblées de Dieu, et en particulier leur "néo-pentecôtisation» (p. 248) progressive, mouvement qui s'est développé par le bas (à l'initiative de jeunes pasteurs ou de fidèles) et qui se marque en particulier par la multiplication des manifestations de l'Esprit Saint (avec un accent plus important mis sur les dons de guérison et de prophétie) et une sensibilité, dans ces milieux (plutôt urbains), pour la doctrine ou la théologie de la prospérité. Emblématique de cette évolution est également le phénomène des "croyants-guérisseurs ", auxquels l'auteur consacre une longue analyse. Apparus vers le milieu des années 1980, en même temps que se banalisaient dans un nombre toujours plus important de paroisses (surtout en milieu urbain) les cultes tournant autour de prières collectives de délivrance, leur développement correspond à l'émergence d'un pôle de légitimité purement charismatique dans une Eglise qui, en dépit de ses fondements pentecôtistes, présente un caractère fortement institutionnalisé. P.-J. Laurent nous décrit en effet comment, depuis une bonne quinzaine d'années, l'autorité des pasteurs est en permanence susceptible, surtout en milieu urbain, de se voir concurrencer par celle d'« évangélistes" ou de «prophètes » (présentant la caractéristique de n'avoir pas fréquenté d'Institut Biblique), qui repose bien davantage que celle des pasteurs sur un charisme personnel. Ces développements sur les relations entre les modes de légitimation de l'autorité religieuse constituent évidemment la reprise d'une question classique de la sociologie religieuse depuis M. Weber, et on peut d'ailleurs s'étonner que l'auteur ne fasse pour ainsi dire aucune allusion aux catégories wébériennes, ni pour les reprendre, ni pour les retravailler ${ }^{2}$. Ceci dit, l'auteur, dans son analyse très fouillée du phénomène des " croyants-guérisseurs ", met parfaitement en évidence les phénomènes de concurrence qui se créent entre croyants-guérisseurs, et entre eux et les pasteurs, autour de la puissance et de la légitimation de leurs dons spirituels, ainsi que l'ambiguïté de leurs positions sur la question délicate de la rémunération (l'offre de guérison divine étant en principe gratuite mais tous les croyants-guérisseurs d'un certain niveau étant des " professionnels » vivant de leur capital religieux). L'auteur montre aussi comment l'offre de guérison des Assemblées de Dieu fonctionne à partir de deux scènes (l'une privée, engageant, dans le cadre d'une séance individualisée de délivrance, le croyant-guérisseur et son "patient", en présence éventuellement de quelques assistants du premier et de quelques proches du second; l'autre publique, constituée par les séances collectives de prières de délivrance), leur articulation et leur mobilisation successive étant au principe de l'efficacité thérapeutique du dispositif.

7 On l'aura compris à travers cette rapide synthèse (qui reste très partielle), l'ouvrage parvient incontestablement à donner une vue d'ensemble du pentecôtisme des Assemblées de Dieu au Burkina Faso, en conjuguant de façon équilibrée analyses plus générales et études de cas ou descriptions fouillées (l'intérêt pour le rituel pentecôtiste et ses ressorts est également tout à l'honneur de l'ouvrage), ancrées dans une très bonne connaissance des sociétés mossi et burkinabée. Quelques réserves quand même avant de discuter ou de prolonger plus spécifiquement la partie historique de l'ouvrage et la présentation du contexte transnational dans lequel évoluent les Assemblées de Dieu du Burkina Faso. 
8 Premièrement, on peut repérer dans cette étude des formes d'imprécisions conceptuelles ; par exemple, lorsque l'auteur construit l'opposition entre les catégories de "concorde coutumière» et de "concorde civile» (auxquelles il a par la suite abondamment recours), il semble qu'il se laisse en quelque sorte prendre au piège de la traduction (dans le sens où il y a probablement abus d'une " catégorie indigène »), quand il soutient que la mise en place d'un espace public, caractéristique de l'ordre de la «concorde civile», suppose l'extension à l'ensemble de la société d'une forme de confiance similaire à celle (kis-sida) qui, dans le monde de la " concorde coutumière ", est censée caractériser les relations à l'intérieur des groupes de parenté (pp. 256-272), et ce, alors que A. Giddens (1994), au moins, a bien montré les transformations de la notion de confiance avec le développement de la modernité. Autre exemple, la spécificité que l'auteur veut réserver au « bricolage social » et la distinction qu'il veut finalement établir entre celui-ci et le «bricolage culturel» (p. 412), dont on voit mal ce qu'elles recouvrent et sur quels types de critères elles reposent. Autre cas encore, l'utilisation fréquente et nullement problématisée du concept de «moi multiple » (l'expression semble provenir, dans le sens où l'utilise l'auteur, de M. Douglas, 1999, à laquelle il ne renvoie jamais explicitement sur ce point), que J.-P. Laurent emploie pour désigner la persistance d'une conception indigène de la personne comme composée de plusieurs entités et susceptible d'être possédée. Or, en sociologie et en anthropologie, au moins depuis les études de Bastide (1970) sur le «principe de coupure» et celle de Bourdieu et Sayad (1964) sur le «dédoublement», il me semble qu'une autre tradition, nettement plus importante, s'efforce de penser la pluralité interne de l'acteur au niveau de la cohabitation le plus souvent non consciente (ou au moins non réfléchie) de logiques (en tant que systèmes de schèmes) différentes, voire contradictoires chez les mêmes individus (voir aussi Bourdieu, 1997 : 190 ; Lahire, 1998). Le caractère multiple du moi renvoie davantage dans cette tradition à la coexistence (envisagée comme plus ou moins génératrice de tensions, et entraînant des phénomènes d'alternance et d'oscillation) de schèmes et non à la multiplicité des composantes spirituelles de la personne. Etablir dans quelle mesure celleci peut correspondre à une forme de reconnaissance du caractère pluriel du soi (cette fois en termes de cohabitation de logiques) pose la question de la transparence des acteurs à eux-mêmes (entre réflexivité et logiques pratiques), n'a rien d'évident même si on voit bien que c'est, au moins en partie, le cas, et aurait probablement mérité une approche plus approfondie de la question du « moi multiple».

Deuxièmement, des aspects non négligeables de l'objet auraient probablement mérité davantage d'attention. Lorsque l'auteur aborde par exemple la question de la « rente » du développement sur laquelle certains pasteurs construisent, à l'instar d'autres big men, un réseau de dépendants (qui, bien entendu, comme le rappelle fréquemment l'auteur, les « obligent » en retour), il laisse complètement dans l'ombre la rente qui peut découler de la position même de pasteur d'une paroisse importante, et dès lors les luttes de position qui peuvent exister pour obtenir, par exemple, une affectation en milieu urbain plutôt qu'en milieu rural ${ }^{3}$. De même, bien que l'auteur évoque souvent les «risques de segmentation" ou les tendances segmentaires caractéristiques des «sectes protestantes ", on peut regretter qu'il ne s'attarde pas un peu plus sur la situation telle qu'elle se présente à cet égard au Burkina Faso : même si le titre renvoie, probablement pour des raisons éditoriales bien compréhensibles, aux " pentecôtistes du Burkina Faso ", il n'est en effet question, d'un bout à l'autre de l'ouvrage, que des Assemblées de Dieu ${ }^{4}$. Celles-ci dominent en effet très largement la scène pentecôtiste burkinabée, puisqu'elles 
regroupent 90 \% environ des pentecôtistes du pays (p. 29). A aucun moment cependant P.J. Laurent ne souligne qu'il s'agit là en Afrique d'une grande spécificité du pentecôtisme burkinabé : une telle domination dénominationnelle correspond pourtant à une situation unique dans les pays d'Afrique subsaharienne où le pentecôtisme a connu des développements importants.

\section{Les Assemblées de Dieu du Burkina Faso en Afrique de l'Ouest}

10 Avec la question de la spécificité du pentecôtisme burkinabé en Afrique de l'Ouest, on touche en fait aux manques de l'ouvrage: l'objet n'est guère situé dans son contexte transnational, et c'est l'un des points sur lesquels je souhaite plus particulièrement rebondir ici. En effet, quelques aspects de l'expansion transnationale et des développements déjà anciens ${ }^{5}$ ou plus récents des Assemblées de Dieu au Burkina Faso et, plus généralement, en Afrique de l'Ouest méritent de retenir davantage l'attention et prolongent utilement l'étude ici présentée.

11 Premier prolongement en effet: dès 1936, un missionnaire américain vient déposer un évangéliste mossi au Nord-Togo ${ }^{6}$, dans la ville presque frontalière de Dapaong, et dès le départ, celui-ci promeut la culture d'un nouveau type de bananiers et amène une nouvelle espèce de cochons (plus gros), qui deviendront vite « les bananiers de la Bonne Nouvelle » et « les porcs de la Bonne Nouvelle », que ceux qui les adoptent commencent à exporter vers le Ghana (alors Côte de l'Or). En encourageant aussi les cultures individuelles auprès des jeunes hommes qui forment les premiers convertis, il s'engage dès le départ dans la promotion d'une forme de modernité villageoise (qu'illustrent également les écoles primaires construites par les missionnaires américains, jusque dans des centres villageois, au Sud-Est du Nigeria, dès 1940) que P.-J. Laurent a bien raison de relever comme ayant été une composante présente pour ainsi dire ab initio de l'action des Assemblées de Dieu en Afrique de l'Ouest. Cette modernité ne se limite vraisemblablement pas à la figure du pasteur-paysan mossi, même si celle-ci en est évidemment à l'avant-garde et si l'ancrage rural des Assemblées de Dieu a été et reste probablement plus important en pays mossi que partout ailleurs en Afrique de l'Ouest, ce qu'il faut évidemment comprendre à la lumière du fait que les missionnaires américains ont là d'emblée commencé à travailler en moore, la langue véhiculaire indigène, ce qui, à ma connaissance, ne s'est reproduit nulle part ailleurs en Afrique de l'Ouest. Par ailleurs, le fait que les missionnaires américains s'exprimaient finalement mieux en moore qu'en français ne facilitait pas toujours (même si l'auteur n'aborde pas la question) les rapports avec les administrateurs coloniaux, surtout lorsque ceux-ci étaient catholiques et, spécialement après la guerre, dans un contexte politique de méfiance envers les stratégies (au moins) imputées aux Américains, que l'on soupçonnait de vouloir s'implanter en Afrique de l'Ouest.

12 Deuxième point qui me semble devoir être souligné (et que n'aborde pas l'ouvrage), il semble que les relations entre les missionnaires américains et leurs auxiliaires africains aient été empreintes de formes de racisme (qui ont découragé certains de ceux-ci), et ce, au moins jusque dans les années 1960. Rappelons-nous d'ailleurs que les Assemblées de Dieu étaient, au début de leur existence, de tendance ségrégationniste. Le pasteur S. $\mathrm{Kalifa}^{7}$, converti dans la région de Dapaong au milieu des années 1940 puis, à partir de 
1965, premier président des $\mathrm{AD}$ du Bénin après leur séparation de celles du Togo, se dit aujourd'hui très content que les missionnaires américains aient apporté la parole de Dieu, mais se souvient aussi que certains au moins abîmaient d'abord les vêtements qu'ils voulaient donner à leurs auxiliaires africains pour que ceux-ci ne puissent pas prétendre se hausser à leur niveau à travers l'habillement, ou encore qu'ils refusaient de partager leur eau. Le pasteur Kalifa a ainsi fini par dire un jour qu'il ne voulait plus qu'un missionnaire l'appelle « frère ». Il a dit cela "sans méchanceté ni orgueil, mais à cause de leur façon de faire avec les Africains », de leur mépris et de leur manque de considération.

Marquées par le racisme et l'anti-intellectualisme, il semble aussi que les missions des AD aient eu, au départ, une position ambivalente envers l'instruction (le risque étant que celle-ci détourne les Africains de la Bible), point que P.-J. Laurent aborde à peine ${ }^{8}$. Le pasteur B. D. Pasgo', converti de la première heure à Dapaong, membre de la première promotion de l'Institut Biblique de Koubri (entrée en 1941, en Haute-Volta) puis premier président des AD du Daho-Togo, se souvient ainsi avoir dû lutter âprement avec certains missionnaires pour pouvoir mettre ses enfants à l'école, et avoir, par ailleurs, plusieurs fois dû faire face à (ou eu à subir) des raisonnements du type «il ne faut pas trop donner aux Africains, sinon ils deviennent orgueilleux ». Les études supérieures des pasteurs africains semblent aussi ne pas avoir été encouragées avant les années 1960 (et, en fait, la création du Cours Supérieur de Théologie de l'Afrique de l'Ouest, à Lomé, en 1970 ${ }^{10}$ ). Au Nigeria, certains missionnaires décourageaient les pasteurs de poursuivre leur formation et n'étaient pas favorables à la construction d'écoles, alors que d'autres, y voyant un outil d'évangélisation, ont promu de telles écoles depuis 1940 (quatre écoles primaires des Assemblées de Dieu avaient été ouvertes au Sud-Est du Nigeria en 1944, et une quinzaine au milieu des années 1950) (General Council of Assemblies of God - Nigeria, 1989 : 49-56).

Troisièmement, il semble que P.-J. Laurent, d'une part, surestime la place que les $\mathrm{AD} d u$ Burkina Faso ont occupée dans l'expansion de l'Eglise en Afrique de l'Ouest et, d'autre part, qu'il distingue mal deux périodes, premièrement celle de l'action missionnaire des quelques premiers pasteurs-évangélistes mossi dans les années 1930-195011, et deuxièmement celle du renouveau de cette action missionnaire à partir de 1984 et la création de la VIMAB (Vision Missionnaire des Assemblées de Dieu du Burkina Faso) ${ }^{12}$.

En effet, en ce qui concerne ce dernier point, l'auteur semble considérer que les Assemblées de Dieu du Burkina ont dès les années 1930 fourni des missionnaires qui se sont dispersés dans l'ensemble de l'Afrique (voir les pp. 29 et 51), et que ceux-ci ont joué un rôle moteur dans l'expansion des Assemblées de Dieu au moins en Afrique de l'Ouest. Or, si le pays mossi a bien été le point de départ principal de l'expansion des Assemblées de Dieu en Afrique de l'Ouest, s'il y a bien eu, dans les années 1930-1950, des évangélistes mossi avec les missionnaires américains au moins au début de l'implantation de l'Eglise dans les régions frontalières du Sud-Burkina ${ }^{13}$ et si, au Togo en particulier, ceux-ci ont même joué un rôle important dans la première évangélisation de l'extrême Nord du pays, il faudrait veiller à ne pas surestimer l'importance de la contribution mossi au travail missionnaire sur lequel s'est, au départ, construite l'expansion des Assemblées de Dieu en Afrique de l'Ouest et, plus largement, en Afrique subsaharienne. Le Togo avait par exemple accueilli, jusqu'à la fin des années 1980, 93 missionnaires résidents, dont cinq Mossi dans les années 1930-1940, puis deux autres (un couple) à partir de 1988, mais tous les autres étaient Américains. De même, le Nigeria, où se trouve la plus importante Eglise nationale des Assemblées de Dieu en Afrique de l'Ouest, avait accueilli, jusqu'en 1989, 91 missionnaires, dont aucun Mossi ni même aucun Africain. Le Bénin avait accueilli, 
jusqu'en 1998, 23 couples de missionnaires américains pour 9 couples burkinabés ${ }^{14}$ et quelques missionnaires d'autres nationalités.

Le deuxième temps de l'action missionnaire des Assemblées de Dieu burkinabées démarre en 1984 avec la création de la VIMAB par un groupe de pasteurs qui regrettent précisément l'absence d'ambition missionnaire de leur Eglise nationale: le premier missionnaire est envoyé au Bénin en $1987^{15}$, et d'autres suivent au Togo, au Niger, au Mali, etc. Mais pour ce qui est de la position que ces missionnaires occupent dans ces différents pays, elle est loin d'être déterminante là où les Assemblées de Dieu sont bien établies et dominantes sur la scène pentecôtiste nationale (comme au Bénin ou au Togo ${ }^{16}$, et ne joue vraiment un rôle que dans les pays à majorité musulmane (comme le Sénégal, le Mali ou le Niger) où les pentecôtistes dans leur ensemble ne forment qu'une minorité très faible, voire presque négligeable. Et là encore, les missionnaires mossi-burkinabés ne sont pas seuls, puisque les Assemblées de Dieu du Bénin, du Togo, de Côte d'Ivoire, du Nigeria et du Ghana ont elles aussi envoyé des missionnaires dans d'autres pays d'Afrique occidentale ${ }^{17}$ et même centrale.

En ce qui concerne en effet la place dominante que les Assemblées de Dieu du Burkina Faso occuperaient en Afrique de l'Ouest, un bref aperçu de la situation de l'Eglise nigériane (dont l'auteur signale cependant qu'elle compte environ deux fois plus de fidèles, pp. 29-30) permettra de compléter rapidement cette relativisation d'une conception trop centrée sur le Burkina Faso. Arrivés en 1939 à Umuahia, près d'Enugu, dans le Sud-Est du pays, suite à une demande adressée aux Assemblées de Dieu des EtatsUnis par une petite Eglise pentecôtiste indigène (The Church of Jesus Christ) qui cherchait une affiliation pentecôtiste occidentale (et dont les fondateurs recevaient déjà depuis cinq ans le périodique des Assemblées de Dieu The Pentecostal Evangel), les premiers missionnaires ont la chance, pour ainsi dire, de « récupérer ", cinq ans après sa fondation, une petite Eglise d'une quinzaine de paroisses, mais déjà implantée (du fait des réseaux migratoires) dans les trois régions administratives que comptera le Nigeria après la deuxième guerre mondiale (Nord, Ouest et Est) (General Council of Assemblies of God Nigeria, 1989: 6-20 et 29-35). En outre, les Assemblées de Dieu du Nigeria bénéficièrent dès leurs premières années d'existence du ralliement de petites Eglises ou d'assemblées locales, ce qui accéléra également leur croissance. Dès 1940, le premier Institut Biblique est créé à Umuahia (son homologue burkinabé avait été créé en 1939). Le deuxième suit dans la province de Bénin en 1949, le troisième au Nord-Nigeria (près de Kaduna) en 1955, le quatrième au Sud-Ouest (à Ibadan) en 1962, et le cinquième à l'Est du pays encore (à Ogoja) en 1969 (idem: 47-53) ${ }^{18}$. Une presse destinée à la diffusion de la littérature de l'Eglise est installée à Aba (à proximité de Port-Harcourt) vers le milieu des années 1940 ( idem: 68), soit à la même époque que celle du Burkina Faso, même si cette dernière semble davantage avoir eu vocation, à partir de 1957, à exporter de la littérature ecclésiale dans les pays voisins (p. 53). Les Assemblées de Dieu du Nigeria obtiennent leur indépendance comme Eglise nationale en 1964, soit, il est vrai, neuf ans plus tard que celles du Burkina Faso et huit ans après celles du Daho-Togo, mais le maillage de leurs Eglises, même s'il est encore large, couvre déjà à cette époque la plus grande partie du territoire national et ses centres régionaux les plus importants. En 1964, on dénombre déjà 600 églises locales, 450 pasteurs et 25.000 fidèles des Assemblées de Dieu au Nigeria ( PE, 19 avril 1964, p. 16), pour 198 églises, 184 pasteurs et 10.000 fidèles au Burkina Faso la même année (PE, 26 janvier 1964, p. 14). En outre, les Assemblées de Dieu du Nigeria ont aujourd'hui en poste plus de 80 missionnaires résidents à l'étranger (principalement au 
Cameroun et au Niger, mais aussi au Gabon, en Guinée équatoriale, en Gambie, au Liberia, au Bénin et aux Etats-Unis - voir, notamment, Ogba, $2002: 69-70$ ), ce qui est évidemment bien davantage que le nombre de missionnaires de la VIMAB (inférieur à une vingtaine). Les données comparatives font souvent défaut et ne sont probablement pas toujours fiables, mais ces quelques exemples suffisent à montrer que les développements des Assemblées de Dieu au Burkina Faso, que P.-J. Laurent a tendance à présenter comme absolument uniques en Afrique de l'Ouest et, a fortiori, en Afrique, ont été concurrencés au moins par les développements de l'Eglise au Nigeria dès les années 1940. Et le cas du Kenya au moins demanderait probablement davantage d'investigations avant que l'on ne puisse affirmer que l'antériorité de l'implantation des Assemblées de Dieu au Burkina Faso s'est véritablement traduite au cours du siècle par un rôle moteur primant, dans l'expansion de celles-ci en Afrique subsaharienne, sur les efforts d'évangélisation d'autres Eglises nationales (dans leurs propres pays et au delà), et ce, sans compter le travail missionnaire considérable de l'Eglise américaine (puis, de façon nettement plus modeste, des Eglises française et suédoise au moins) qui a lancé dans la grande majorité des pays les dynamiques qui se sont ensuite indigénisées pour devenir, à mon sens, les facteurs principaux de la croissance de l'Eglise partout où celle-ci a connu des développements importants.

Enfin, on peut regretter que P.-J. Laurent ne situe pas davantage les Assemblées de Dieu du Burkina Faso dans leur contexte transnational au moins africain. Il reste en effet notamment silencieux sur l'Alliance des Assemblées de Dieu d'Afrique, qui existe depuis une dizaine d'années environ (et a probablement été créée dans la foulée de la World Assemblies of God Fellowship, qui voit le jour en 1989). Celle-ci regroupe les différentes Eglises nationales du continent et le président des $\mathrm{AD}$ du Burkina Faso en est actuellement le quatrième président en exercice. Pas un mot non plus sur la Decade of Harvest proclamée dans les Assemblées de Dieu du monde entier (et même au-delà, de façon interdénominationnelle) à partir de 1990 et qui a vu, en Afrique, le nombre de lieux de culte de l'Eglise passer de 11.688 à 24.755 entre 1989 et 1999, tandis que le nombre de " membres et adhérents » passait de 2.140.202 à 8.077.333 pendant la même période ${ }^{19}$. Or, dans les années 1990, c'est évidemment aussi dans le contexte "interne » de ce projet (en plus du contexte "externe » économique et social africain) et dans le cadre d'une politique d'évangélisation intensive aux objectifs pré-définis que s'inscrit la croissance des Assemblées de Dieu du Burkina Faso. Une seule et furtive allusion est faite à une conférence panafricaine des Assemblées de Dieu d'Afrique de l'Ouest en 1982 (p. 64), alors que de tels événements ont eu lieu régulièrement à partir de 1964 et que c'est précisément dans le cadre de tels rassemblements que des projets comme la Decade of Harvest sont relayés.

En un mot, il semble que P.-J. Laurent surestime la place prise par les Assemblées de Dieu du Burkina Faso en Afrique subsaharienne, et même en Afrique de l'Ouest. A force de vouloir rendre justice à la contribution mossi au travail missionnaire de l'Eglise en Afrique occidentale, on en arrive presque à occulter l'importance des flux missionnaires principaux (P.-J. Laurent ne rapporte pas, par exemple, le nombre de missionnaires américains et français ayant résidé au Burkina Faso depuis les débuts de l'Eglise) et celle, majeure, des multiples dynamiques « indigènes » qui, dans chaque pays où les Assemblées de Dieu ont aujourd'hui une Eglise nationale importante, ont rapidement fourni l'essentiel des pasteurs et des évangélistes. Bref, si l'ouvrage présente une analyse fouillée de nombreux aspects du pentecôtisme burkinabé et, plus largement, africain 
contemporain, on aurait souhaité également une mise en contexte plus large (historique, transnationale) de l'objet étudié, qui en aurait incontestablement fourni des clefs de compréhension supplémentaires.

\section{BIBLIOGRAPHIE}

AKIBO, P. K., 1998, Les fruits de la pentecôte. Histoire de l'Eglise Evangélique des Assemblées de Dieu du Bénin, 1945-1998, Abomey-Calavi : EEAD-Bénin.

Assemblées de Dieu du Togo , s.d. Assemblées de Dieu du Togo en 50 ans. History of the Togo Assemblies of God.

BASTIDE, R., 1970, Le prochain et le lointain, Paris : Cujas.

BOURDIEU, P., 1997, Méditations pascaliennes, Paris : Seuil.

BOURDIEU, P. et SAYAD, A., 1964, Le déracinement. La crise de l'agriculture traditionnelle en Algérie,

Paris : Minuit.

DOUGLAS, M., 1999, Comment pensent les institutions, suivi de Il n'y a pas de don gratuit et La connaissance de soi, Paris : La Découverte/MAUSS.

General Council of Assemblies of God - Nigeria, 1989, The Growth of Assemblies of God in Nigeria, Aba : Assemblies of God Press and Bookshop.

GIDDENS, A., 1994, Les conséquences de la modernité, Paris : L'Harmattan.

LAHIRE, B., 1998, L'homme pluriel. Les ressorts de l'action, Paris : Nathan.

OGBA, Rev. A. E., 2002, « Missions Director's Quadriennal Report 1998-2001 », inGeneral Council

Meeting of Assemblies of God Nigeria, pp. 68-72. Polycopié.

WILLAIME, J.-P., 1992, La précarité protestante. Sociologie du protestantisme contemporain, Genève :

Labor et Fides.

\section{NOTES}

1. Aux pasteurs B. Pasgo et S. Kalifa, qui ont accepté de partager avec moi un peu de leurs souvenirs. Par ailleurs, je voudrais aussi remercier ici l'IFRA-Ibadan, qui a financé une grosse partie des recherches sur lesquelles repose cette note critique dans le cadre de son programme «Réseaux transnationaux et nouveaux acteurs religieux en Afrique de l'Ouest ».

2. On voit bien, pourtant, comment, avec le phénomène des «croyants-guérisseurs ", dans une Eglise fortement institutionnalisée comme les $\mathrm{AD}$, les détenteurs des « charismes personnels » les plus importants ne sont pas les plus hauts responsables de la hiérarchie de l'Eglise, dont le charisme se rapproche davantage des charismes «idéologique " (voir Willaime, 1992:15-29) et " de fonction ", même s'il possède aussi inévitablement une dimension " personnelle » puisque la vocation de pasteur repose ici, au départ, sur un appel divin personnel. 
3. Je fais ici référence à un type de situation qui m'a été rapporté comme classique dans les $\mathrm{AD}$ du Togo. Le souci d'une répartition plus équitable des ressources de l'Eglise et de la révision des disparités salariales entre les différentes catégories de pasteurs apparaît aussi, il y a une quinzaine d'années déjà, dans un document émanant des AD du Nigeria (General Council of Assemblies of God - Nigeria, 1989 : 126). Mais, d'une manière générale, l'auteur accorde très peu d'attention aux jeux de pouvoir qui entourent la gestion de l'argent des paroisses, et aux tensions que cela peut générer, par exemple entre le pasteur et le comité paroissial.

4. Rien n'est dit, en particulier, de la Fédération des Eglises et Missions Evangéliques, dont P.-J. Laurent mentionne seulement l'existence (p. 61). Fondée en 1961, elle regroupe pourtant aujourd'hui 17 Eglises évangéliques et pentecôtistes (voir la page web www.cvktv.org/ partners.html\#part).

5. Une source historique de premier plan est ignorée par l'auteur à cet égard, à savoir le périodique des $\mathrm{AD}$, The Pentecostal Evangel (auquel je renvoie systématiquement, dans la suite de ce texte, par PE), qui est entièrement disponible au format PDF, sur internet (moyennant enregistrement du chercheur), sur le site du centre de recherches historiques des AD (Flower Pentecostal Heritage Center, http://ifphc.org/).

6. L'introduction des $\mathrm{AD}$ au Togo et au Bénin remonte en effet à 1936 dans le premier cas et à 1945 dans le second (avec, ici, réception de l'autorisation officielle en 1947), et non l'inverse, comme l'écrit l'auteur à la suite d'une malencontreuse confusion (p. 55).

7. Entretiens réalisés en avril 2002 et en mars 2003 à Calavi, dans la banlieue de Cotonou.

8. Il signale seulement que les missionnaires cherchaient surtout à développer les «aptitudes à l'écriture et à la lecture » des premiers paysans mossi convertis (p. 51), sans relever comme marquant, par exemple, le fait que la première école primaire des $\mathrm{AD}$ au Burkina est construite seulement par les premiers missionnaires français, après 1948 (ibid.).

9. Entretien réalisé en février 2003 à Pagala-gare, au centre du Togo. Le pasteur B. D. Pasgo (et non D. Pasco, comme l'écrit l'auteur quand il en fait brièvement mention, p. 56) est mossi mais établi dès avant sa conversion au Nord-Togo.

10. En prenant progressivement de l'importance, celui-ci deviendra l'ESTAO (Ecole Supérieure de Théologie de l'Afrique de l'Ouest) puis la FATAD (Faculté de Théologie des Assemblées de Dieu). Dès le départ, on y dispense des formations en anglais et en français. Les pasteurs qui y sont formés, venus de l'ensemble de l'Afrique de l'Ouest et même d'Afrique centrale, constituent ensuite l'élite de leurs Eglises nationales respectives. Il y a donc bien eu là une plaque tournante dans la formation des élites ecclésiales, et le Togo a également accueilli, en 1982 et 1986, deux autres bureaux régionaux (pour l'Afrique de l'Ouest) des $\mathrm{AD}$, respectivement celui de l'ICI (Institut par Correspondance Internationale) et celui de l'ALM (Africa Literature Ministries) (Assemblées de Dieu du Togo, s.d.: 14), ce qui a encore renforcé sa dimension de plaque tournante. Au début des années 1990, les formations dispensées par la FATAD ont été décentralisées (les enseignants se déplaçant maintenant vers leurs publics), ce qui a permis d'augmenter beaucoup le nombre d'étudiants. Cinq facultés de théologie des AD délivrent aujourd'hui, en Afrique, une maîtrise en théologie : celles basées au Togo, au Nigeria (où deux autres facultés délivrent aussi des licences), en Afrique du Sud, au Kenya et au Malawi.

11. L'auteur n'aborde pas le fait que les missionnaires américains ont encouragé la formation de Mossi notamment parce qu'ils considéraient que les missionnaires français, trop peu nombreux, ne parviendraient jamais à couvrir l'ensemble de l'Afrique occidentale francophone (voir PE, 4 janvier 1947, p. 8 ; PE, 12 mars 1949, p. 9).

12. Par exemple lorsqu'il affirme (et les phrases qui suivent n'ont rien d'un extrait décontextualisé dont le ton trancherait avec les pages suivantes) de façon un peu rapide, nous le verrons, à la première page du chapitre historique de l'ouvrage que «l'Eglise des Assemblées de Dieu du Burkina Faso a joué un rôle important dans l'expansion du mouvement pentecôtiste sur le continent africain. Il est fréquent de rencontrer aujourd'hui des pasteurs mossi, à la tête de 
missions installées dans différents pays du continent. De même, l'influence des Assemblées de Dieu du Burkina Faso sur les Eglises sœurs d'Afrique est notoire » (p. 29).

13. La façon dont l'auteur s'exprime lorsqu'il évoque l'expansion des $A D$ vers les pays de la Côte du Golfe de Guinée est susceptible d'induire en erreur et de mener à une certaine confusion. P.-J. Laurent affirme en effet que « dès 1936, des prédicateurs mossi ouvraient l'église de Dapaongo, au Bénin et un peu plus tard à Accra au Ghana, suivant, en cela, les chemins traditionnels des migrations mossi vers les pays côtiers. En 1947, les Assemblées de Dieu s'installèrent au Togo. L'Eglise de Côte d'Ivoire fut fondée par des pasteurs missionnaires mossi » (p. 55). Outre la confusion entre les dates de pénétration de l'Eglise au Bénin et au Togo (voir supra), l'auteur semble en effet confondre ici deux situations, qui sont celles du Togo, du Bénin et du Ghana d'une part, et de la Côte d'Ivoire d'autre part. En effet, au Togo (en 1936) et au Bénin (en 1945), l'arrivée des $\mathrm{AD}$ correspond à un simple passage de la frontière, et l'Eglise restera confinée dans la moitié Nord du pays jusqu'à l'indépendance par des accords signés avec d'autres dénominations dans le cadre de la Fédération des Eglises Protestantes d'Afrique de l'Ouest : il ne saurait dès lors être question ici d'une reprise par les $\mathrm{AD}$ des «chemins traditionnels des migrations mossi vers les pays côtiers ». Au Ghana (en 1931-32), l'Eglise s'installe au cœur du Nord du pays (PE, 7 mai 1932, p.15). C'est en Côte d'Ivoire (en 1937-1938) que les AD s'installent par contre d'emblée au Sud du pays, redoublant clairement donc, là, un itinéraire migratoire mossi habituel.

14. Cinq de ces couples renvoient cependant à des étudiants formés au Nord-Dahomey même, par l'Institut Biblique de Natitingou, à partir de 1949, puis envoyés un moment sur le terrain dans la région (voir la note 11). Deux couples correspondent à des missionnaires envoyés au NordDahomey dans les années 1950, et les deux derniers enfin aux deux couples qui se sont succédés au Sud-Bénin depuis 1987.

15. Il y avait déjà à cette époque quelques missionnaires burkinabés en Afrique de l'Ouest, mais ceux-ci n'étaient pas supportés financièrement par leur Eglise nationale, n'étaient pas coordonnés et, pour tout dire, étaient là à titre individuel.

16. Au Bénin, le missionnaire burkinabé est en charge de l'une des quelques 650 églises locales du pays, tandis qu'au Togo, le missionnaire burkinabé n'est même pas associé directement à l'Eglise nationale des $\mathrm{AD}$ mais enseigne à la FATAD (voir supra, note 10).

17. Le Niger constitue un cas exemplaire de pays où des efforts missionnaires importants sont déployés, puisque six missions des $\mathrm{AD}$ y sont actuellement présentes : celles du Burkina Faso, du Nigeria, de Côte d'Ivoire, de France, des Etats-Unis et de Suède.

18. Par comparaison, le deuxième Institut Biblique du Burkina Faso est ouvert à Ouagadougou (c'est-à-dire à $35 \mathrm{~km}$ du premier, basé à Koubri) en 1965 seulement (p. 57). Un troisième Institut Biblique voit le jour en 1981 (p. 62), alors que les sixième et septième Instituts Bibliques du Nigeria sont ouverts en 1980 et 1982, toujours dans le Sud-Est du pays (General Council..., 1989 : 54).

19. Ces chiffres sont évidemment ceux de l'Eglise, les seuls disponibles (voir: http:// www.africawatch.com/stats.htm). 


\section{AUTEUR}

\section{JOËL NORET}

Joël Noret est aspirant du FNRS et doctorant en anthropologie au Centre d'Anthropologie Culturelle (CAC) de l'Université Libre de Bruxelles et au Centre d'Etudes Africaines (CEAf) de l'Ecole des Hautes Etudes en Sciences Sociales. Il poursuit actuellement ses recherches sur les funérailles et la place des morts au Sud-Bénin ainsi que sur l'expansion des Eglises pentecôtistes dans la même région et sur le développement des Eglises africaines en Belgique. 